\title{
Tensor-Product Representation for Switched Linear Systems
}

\author{
Dengyin Jiang, Lisheng Hu \\ Department of Automation, Shanghai Jiao Tong University, Shanghai, China \\ Email: Ishu@sjtu.edu.cn
}

Received 8 February 2015; accepted 25 March 2015; published 30 March 2015

Copyright (C) 2015 by authors and Scientific Research Publishing Inc.

This work is licensed under the Creative Commons Attribution International License (CC BY). http://creativecommons.org/licenses/by/4.0/

c) (i) Open Access

\begin{abstract}
This paper presents the method for the construction of tensor-product representation for multivariate switched linear systems, based on a suitable tensor-product representation of vectors and matrices. We obtain a representation theorem for multivariate switched linear systems. The stability properties of the tensor-product representation are investigated in depth, achieving the important result that any stable switched systems can be constructed into a stable tensor-product representation of finite dimension. It is shown that the tensor-product representation provides a high level framework for describing the dynamic behavior. The interpretation of expressions within the tensor-product representation framework leads to enhanced conceptual and physical understanding of switched linear systems dynamic behavior.
\end{abstract}

\section{Keywords}

\section{Switched Linear Systems, Tensor-Product Representation, Stability}

\section{Introduction}

Switched systems as an important class of hybrid systems [1] have drawn considerable attention in recent years. This is mainly due to the switched and hybrid nature of many physical processes and the growing use of computers in the control of physical plants [2] [3]. And, many dynamical systems encountered in engineering practice exhibit switching between several subsystems that is dependent on various environmental factors, for example, sampled-data control systems [4]-[9], Markov jump systems [10]-[12], and references therein. The extensive engineering applications of switched systems are also motivated by the better performance that can be achieved using a controller switching strategy [13] [14]. Currently, there is enormous growth in the literature in the study of switched systems [15]-[17]. These dynamical systems combine behaviors that are typical of continuous-time dynamical systems with behaviors that are typical of discrete-time dynamical systems. Hybrid systems [1], in 
which continuous dynamics and discrete events coexist, are considered to be a natural modeling framework for switched systems. Thus, a switched system is a dynamical system that consists of a finite number of subsystems described by differential or difference equations and a logical rule that orchestrates switching between these subsystems [2] [15] [16] [18]-[20].

On the other hand, switched systems cannot be represented in the framework of linear operators due to the logical switching space. For multivariate systems, the relationship between the rows vectors of the matrix space and that between the column vectors might be important for finding a projection to represent the systems operator. Motivated by this idea, switched systems may be represented in the framework of multi-linear algebra. Multilinear algebra, the algebra of higher-order tensors, may be applied for analyzing the multi-factor structure of switched systems, including input space and logical switching space. Based on the considerations of multi-linear algebra, tensor-product representation is used to investigate the switched systems expression. From the theoretical point of view, this approach extended the subspace method using matrix technique to the multivariate switched systems. The multilinear algebra, the algebra of higher-order tensors (also being a multidimensional or Nway array), which are the higher-order equivalents of vectors (first order) and matrices (second order), i.e., quantities of which the elements are addressed by more than two indices, may be applied for analyzing the multifactor structure of multivariate switched systems or such class of nonlinear systems.

In this paper, we investigated the more general problem of tensor-product representations for multivariate switched linear systems. We obtain the tensor-product representation theorem for globally table or globally asymptotically stable multivariate switched linear systems which are only defined on subspaces of algebras. This generalization allows us to obtain a Hahn-Banach extension theorem for such maps, which leads to further results on the Haagerup tensor-product norm applied into more general class of nonlinear systems. The stability properties of the tensor-product representation are investigated in depth, which any stable switched system admits a stable tensor-product representation of finite dimension. It is shown that the tensor-product representation provides a high level framework for describing the dynamic behavior of switched systems. The interpretation of expressions within the tensor-product representation framework leads to enhanced conceptual and physical understanding of switched linear systems dynamic behavior.

The main contribution of this paper is to obtain a general expression of tensor-product representation for multivariate switched systems, and the tensor-product representation is conceptually simple and relatively easy to express on the Haagerup tensor-product norm for multivariate switched systems. We focus on the correspondence between completely bounded multilinear maps and completely bounded linear maps on tensor products endowed with the Haagerup norm [21]-[23]. An abstract characterization of operator systems allows us to embed these tensor product spaces into $C^{*}$-algebras, after which the techniques developed in [24] lead to the extension and representation theorem. The idea of matrix multiplication spread from the definition of a completely bounded multilinear operator to the Haagerup tensor product and matricial operator spaces has implemented, see [25] [26] for a detailed discussion of completely bounded linear operators, and [22] [27] for the multilinear case. And more details can be founded in related review paper [28].

The rest of this work is organized as follows. Section 2 contains the preliminaries on the tensor-product of vectors and matrices and the basic algebraic tools are provided. Section 3 gives the problem formulation for multivariate switched linear systems expression and its relation with bilinear algebra expression. In Section 4, the tensor-product representation for multivariate switched linear systems are investigated, and the stability properties of the tensor-product representation based on the proposed methods are also investigated, which is followed by concluding conclusion in Section 5 .

Notations: The notations throughout this paper are standard. $\mathbf{R}^{n}$ denotes the $n$ dimensional Euclidean space. $\mathbf{R}^{n \times m}$ is the set of all $n \times m$ real matrices. G denotes the systems function. $I$ is the identity matrix with appropriate dimensions, $\langle\cdot, \cdot\rangle$ is the inner product, and $\|\cdot\|_{h}$ is the Haagerup norm. The symbol $\oplus$ denotes the direct sum of vectors or spaces, as well as $\odot$ and $\otimes$ denote the matching elementwise Kronecker product and Kronecker product, respectively. And, tensors are written as calligraphic letters $(\mathcal{X}, \mathcal{Y}, \cdots)$.

\section{Preliminaries}

In this section, we give some necessary preliminaries on the tensor-product of vectors and matrices, mainly concerning with basic algebraic tools on definitions and the correspondence between completely bounded bilinear maps and completely bounded linear maps on Haagerup tensor products norm, which will be used in this 
paper. And, the $C^{*}$-algebraic technique is used to investigate the tensor-product representation of multivariate switched linear systems.

Let $\mathbf{A}$ and $\mathbf{B}$ denote unital $C^{*}$ algebras and $\mathbf{E}$ and $\mathbf{F}$ will denote subspaces. $\mathbf{E}^{*}$ and $\mathbf{F}^{*}$ will denote the subspaces consisting of the adjoints of elements in $\mathbf{E}$ and $\mathbf{F}$, respectively. The Haagerup norm on the algebraic tensor product $\mathbf{E} \otimes \mathbf{F}$ is defined as follows,

$$
\|u\|_{h}=\inf \left\{\left\|\sum_{i=1}^{n} e_{i} e_{i}^{*}\right\|^{1 / 2}\left\|\sum_{i=1}^{n} f_{i}^{*} f_{i}\right\|^{1 / 2}\right\}
$$

where the infimum is taken over all possible representations $u=\sum_{i=1}^{n} e_{i} \otimes f_{i}$. The resulting normed space is written $\mathbf{E} \otimes_{h} \mathbf{F}$. It is clear from the definitions that $\mathbf{E} \otimes_{h} \mathbf{F}$ is a matrix normed space in the sense of the reference [29].

Let $M_{n}(\mathbf{A})$ denote the $C^{*}$-algebra of $n \times n$ matrices over the field of complex numbers. If $\mathbf{V}$ is a vector space then the space $M_{n}(\mathbf{V})$ of $n \times n$ matrices over $\mathbf{V}$ is naturally a vector space; indeed $M_{n}(\mathbf{V})$ is a bimodule over the ring $M_{n}(\mathbf{A})$. If $x \in M_{n}(\mathbf{V})$ and $y \in M_{n}(\mathbf{V})$ then $x \oplus y$ denotes the matrix in $M_{(n+m)}(\mathbf{V})$ with $x$ and $y$ in the diagonal blocks (in that order) and zero in the off-diagonal blocks.

Definition 1 [30] Let $A=\left(x_{i j}\right)$ be in $M_{n, k}(\mathbf{A})$ and $B=\left(y_{i j}\right)$ be in $M_{k, m}(\mathbf{B})$; then $A \odot B$ is the element of $M_{n, m}(\mathbf{A} \otimes \mathbf{B})$ given by

$$
A \odot B=\left[\sum_{r=1}^{k} x_{i r} \otimes y_{r j}\right]
$$

It is valuable to note that if $L$ is a matrix of scalars, then $A \odot(L B)=(A L) \odot B$.

If $\mathbf{X}$ and $\mathbf{Y}$ are matrix normed spaces then we define the Haagerup matrix norm on $\mathbf{X} \otimes \mathbf{Y}$ by setting for $U \in M_{n}(\mathbf{X} \otimes \mathbf{Y})$,

$$
\|U\|_{h}=\inf \left\{\sum_{k=1}^{m}\left\|A_{k}\right\|\left\|B_{k}\right\|: U=\sum_{k=1}^{m} A_{k} \odot B_{k}\right\}
$$

where the infimum is taken over all such expressions for $U$ with $A_{k}$ in $M_{n, n_{k}}(\mathbf{X}), B_{k}$ in $M_{n_{k}, n}(\mathbf{Y})$. It is not difficult to see that with these definitions $\mathbf{X} \otimes \mathbf{Y}$ becomes a matrix normed space, which we denote $\mathbf{X} \otimes_{h} \mathbf{Y}$.

Definition 2 [28] Let $\mathbf{A}$ and $\mathbf{B}$ be $C^{*}$-algebras, and let $\phi: \mathbf{A} \rightarrow \mathbf{B}$ be a linear operator. Define $\phi_{n}=\phi \otimes I_{n}$ from $\mathbf{A} \otimes M_{n}$ into $\mathbf{B} \otimes M_{n}$, where $I_{n}$ is the identity operator on $M_{n}$, for each $n \in N$; that is,

$\phi_{n}\left(a_{i j}\right)=\left[\phi\left(a_{i j}\right)\right]$ for all $\left(a_{i j}\right) \in M_{n}(\mathbf{A})$. An operator $\phi$ is completely bounded if $\left\{\left\|\phi_{n}\right\|: n \in N\right\}$ is bounded, and the completely bounded norm $\|\cdot\|_{c b}$ is defined by

$$
\|\phi\|_{c b}=\sup \left\{\left\|\phi_{n}\right\|: n \in N\right\}
$$

Proposition 1 [28] Let $\left(\mathbf{V},\|\cdot\|_{n}\right)$ be a matricial normed space. Then $\mathbf{V}$ is completely isometrically isomorphic to a matricial operator space if and only if $\|v \oplus w\|_{m+n}=\max \left\{\|v\|_{m},\|w\|_{n}\right\}$ for all $v \in M_{m}(\mathbf{V})$, $w \in M_{n}(\mathbf{V})$ and $m, n \in N$.

Let $\mathbf{B}(\mathbf{H})$ denote the bounded linear operators on a Hilbert space $\mathbf{H}$. If $\phi: \mathbf{E} \otimes_{h} \mathbf{F} \rightarrow \mathbf{B}(\mathbf{H})$ is a linear map, then linear maps $\phi_{n}: M_{n}\left(\mathbf{E} \otimes_{h} \mathbf{F}\right) \rightarrow M_{n}(\mathbf{B}(\mathbf{H}))$ can be defined, for each positive integer $n$, by $\phi_{n}\left(u_{i j}\right)=\left[\phi\left(u_{i j}\right)\right]$. If $V: \mathbf{E} \times \mathbf{F} \rightarrow \mathbf{B}(\mathbf{H})$ is a bilinear map then $V$ is defined to be the smallest constant $K$ satisfying $\|V(e, f)\| \leq K\|e\|\|f\|$. Any bilinear map $V: \mathbf{E} \times \mathbf{F} \rightarrow \mathbf{B}(\mathbf{H})$ induces bilinear maps $V_{n}: M_{n}(\mathbf{E}) \times M_{n}(\mathbf{F}) \rightarrow M_{n}(\mathbf{B}(\mathbf{H}))$ where the $(r, s)$ entry of $V_{n}\left(\left(e_{i j}\right),\left(f_{i j}\right)\right)$ is

$$
V_{n}\left(\left(e_{i j}\right),\left(f_{i j}\right)\right)_{(r, s)}=\sum_{k=1}^{n} V\left(e_{r k}, f_{k s}\right)
$$

It is easiest to visualize this as formal matrix multiplication of $\left(e_{i j}\right)$ and $\left(f_{i j}\right)$. If $\sup _{n}\left\|V_{n}\right\|$ is finite then we say that $V$ is completely bounded and $\|V\|_{c b}$ will denote this supremum. These definitions were first given in [27].

Lemma 1 [22] The Haagerup norm on $M_{n}(\mathbf{E} \otimes \mathbf{F})$ is equal to the quotient norm induced by the Haagerup norm on $M_{n}(\mathbf{E}) \otimes M_{n}(\mathbf{F})$.

Proposition 2 [22] Let $V: \mathbf{E} \times \mathbf{F} \rightarrow \mathbf{B}(\mathbf{H})$ be a bilinear map and let $\phi: \mathbf{E} \otimes \mathbf{F} \rightarrow \mathbf{B}(\mathbf{H})$ be the associated 
linear map. Then $V$ is completely bounded if and only if $\phi$ is completely bounded and $\|V\|_{c b}=\|\phi\|_{c b}$.

\section{Problem Formulation}

We consider a collection of discrete-time linear systems described by the equations with parametric uncertainties of process transfer functions and disturbance transfer functions with a class of piecewise constant functions, $\sigma: \mathbf{R}^{+} \rightarrow S$, which serves as the switching signal between the collection of discrete-time systems (6) given as

$$
y_{t}=G_{\sigma}\left(z^{-1}\right) u_{t}+H_{\sigma}\left(z^{-1}\right) a_{t}
$$

where $u_{t} \in \mathbf{R}^{p}$ is the input vector, $y_{t} \in \mathbf{R}^{m}$ is the output vector, $G_{\sigma}\left(z^{-1}\right): \mathbf{R}^{p} \rightarrow \mathbf{R}^{m}$ is a family of discrete process transfer functions that is parametrized by some index set $S, a_{t} \in \mathbf{R}^{q}$ is the Gaussian distributed external disturbance that is a zero mean white noise satisfying, $E\left\{a_{t}\right\}=0, E\left\{a_{t} a_{t}^{\mathrm{T}}\right\}=\rho$, where $\rho$ is a positive definite diagonal matrix, $H_{\sigma}\left(z^{-1}\right): \mathbf{R}^{q} \rightarrow \mathbf{R}^{m}$ is a family of disturbance transfer functions that also is parametrized by some index set $S$. And $\sigma$ denotes the switching signal taking value from the finite index set

$S=\{1,2, \cdots, s\}$, and $s$ represents the integer of the all finite switched subsystems of switched systems and $S$ is the set of positive integers. Furthermore, the switching signal is assumed to be generated by

$$
\sigma(t)=\delta\left(y_{t}\right)
$$

where $\delta: \mathbf{R}^{m} \rightarrow S$. In other words, the discrete mode is determined by a form of static output feedback, and the parametric uncertainty is assumed to be piecewise constant time-varying. The logical switching signal $\sigma$ may be determined by the systems output signals.

It is assumed that the multivariate switched linear systems is globally table or globally asymptotically stable. This assumption essentially is to guarantee the entire switched systems are completely bounded, which is the very key condition for our study here. For this assumption, an interesting question is: under what conditions it is possible to transform the switched linear system in (6) into descriptive multilinear systems framework and further develop the tensor-product representation theorem. It is also desirable that the tensor-product representation property should promote efficiently numerical computation with respect to the multidimensional time series data analysis for estimating the controller performance of multivariate switched linear systems. The main goal of this paper is to obtain a necessary and sufficient condition for the existence of tensor-product representation for (6) based on such multilinear algebra framework. The problem can be formulated as follows.

Problem 1 Given the switched linear system described in (6), derive necessary and sufficient conditions for tensor-product representation of systems operators, which show that the algebraic structure of switched linear systems can abstractly represented in tensor space.

In order to let the developed results focus on the tensor-product representation framework of multivariate switched linear systems in this paper, we consider the simplified systems description form with neglecting the effects of disturbances $\mathbf{a}_{t}$ in the following,

$$
y_{t}=G_{\sigma}\left(z^{-1}\right) u_{t}
$$

Then, we can obtain the switched linear systems operator $G_{\sigma}$, and the equivalent bilinear expression $\mathbf{V}$ is given as

$$
\mathbf{V}: \mathbf{U} \times \mathbf{L} \rightarrow \mathbf{Y} \times \mathbf{L}
$$

where $\mathbf{U}$ and $\mathbf{Y}$ represent the inputs space and outputs space of subsystems, respectively, and the $\mathbf{L}$ represents the logical switching space. And the logic switching signals function $\delta$ in (7) can be expressed as

$$
T: \mathbf{Y} \rightarrow \mathbf{L}
$$

where $T$ denotes the logical operators with the same meaning in (7). From the expression (10), we can see that there exists a map from the switched linear systems outputs to the switching signals. Obviously, the expression (9) exactly describes the map of multivariate switched linear systems from bilinear algebra framework. The systems operator description in (8) can be interpreted as dynamical systems behavior in the physical processes, and the equivalent bilinear form in (9) is expressed from the point of view of algebra structure, which is suitable for the technical development of tensor-product representation approach for controller performance measure of multivariate switched linear systems or such more complex class of multivariate nonlinear systems. 


\section{Tensor-Product Representations}

In this section, we discuss the tensor-product representation of multivariate switched linear systems from the point of view of systems operator by employing the theory of multilinear algebra. We first give a representation theorem for complete bounded linear operator on $\mathbf{E} \otimes_{h} \mathbf{F}$.

Theorem 3 [22] If $\phi: \mathbf{E} \otimes_{h} \mathbf{F} \rightarrow \mathbf{B}(\mathbf{H})$ is completely bounded then there exist representations $\pi: \mathbf{A} \rightarrow \mathbf{B}\left(\mathbf{H}_{1}\right)$, $\rho: \mathbf{B} \rightarrow \mathbf{B}\left(\mathbf{H}_{2}\right)$, isometries $V_{i}: \mathbf{H} \rightarrow \mathbf{H}_{i}$, and a contraction $T: \mathbf{H}_{2} \rightarrow \mathbf{H}_{1}$ such that on elementary tensors

$$
\phi(e \otimes f)=V_{1}^{*} \pi(e) T \rho(f) V_{2}
$$

where $e \in \mathbf{E}, f \in \mathbf{F}$.

Corollary 1 If $V: \mathbf{E} \times \mathbf{F} \rightarrow \mathbf{B}(\mathbf{H})$ is a completely bounded bilinear map then there exist representations $\pi: \mathbf{A} \rightarrow \mathbf{B}\left(\mathbf{H}_{1}\right), \quad \rho: \mathbf{B} \rightarrow \mathbf{B}\left(\mathbf{H}_{2}\right)$, and contractions $V_{i}: \mathbf{H} \rightarrow \mathbf{H}_{i}, \quad T: \mathbf{H}_{2} \rightarrow \mathbf{H}_{1}$ such that

$$
V(e, f)=V_{1}^{*} \pi(e) T \rho(f) V_{2}
$$

Proof. Combine Proposition 2 with Theorem 3, the result is followed.

Now, we focus on studying the tensor-product representation of completely bounded bilinear operators from $\mathbf{A} \times \mathbf{B}$ into $\mathbf{B}(\mathbf{H})$. Let $f$ and $g$ be linear functional from $\mathbf{A}$ into $\mathbf{B}(\mathbf{H})$ and $\mathbf{B}$ into $\mathbf{B}(\mathbf{H})$, respectively. We define a linear function $f \otimes g: \mathbf{A} \otimes \mathbf{B} \rightarrow \mathbf{B}(\mathbf{H})$ by

$$
(f \otimes g)\left(\sum_{i=1}^{r} a_{i} \otimes b_{i}\right)=\sum_{i=1}^{r} f\left(a_{i}\right) g\left(b_{i}\right)
$$

for all $a_{i} \in \mathbf{A}, \quad b_{i} \in \mathbf{B}, \quad i=1,2, \cdots, r$. Let $F=\left[f_{i j}\right]$ and $G=\left[g_{i j}\right]$ be linear operators from $M_{n}(\mathbf{A})$ into $M_{n}(\mathbf{B}(\mathbf{H}))$ and $M_{n}(\mathbf{B})$ into $M_{n}(\mathbf{B}(\mathbf{H}))$, respectively. Then we can define a linear operator $F \odot G: M_{n}(\mathbf{A}) \odot M_{n}(\mathbf{B}) \rightarrow M_{n}(\mathbf{B}(\mathbf{H}))$ by

$$
F \odot G=\left[\sum_{k=1}^{n} f_{i k} \otimes g_{k j}\right]
$$

For a linear operator $F=\left[f_{i j}\right]: M_{n}(\mathbf{A}) \rightarrow M_{n}(\mathbf{B}(\mathbf{H}))$, the adjoint linear operator $F^{*}$ of $F$ can be written as $F^{*}=\left[f_{j i}^{*}\right]$. The linear operator $F$ is completely bounded if and only if each $f_{i j}$ is a completely bounded linear functional on $M_{n}(\mathbf{A})$. If $F$ is completely bounded, then so is $F^{*}$ with $\left\|F^{*}\right\|_{c b}^{i j}=\|F\|_{c b}$.

Let $F=\left[f_{i j}\right]: M_{n}(\mathbf{A}) \rightarrow M_{n}(\mathbf{B}(\mathbf{H}))$ be a completely bounded linear operator and $G=F^{*}$. For all $A_{j}=\left[a_{i_{j}} a_{i_{j+1}}\right] \in M_{m}(\mathbf{A}),(1 \leq j \leq 2)$, we have

$$
\begin{aligned}
(F \odot G)_{m}\left(A_{1}, A_{2}\right) & =\left[\sum_{i_{2}=1}^{m}(F \odot G)\left(a_{i_{1} i_{2}}, a_{i_{2} i_{3}}\right)\right] \\
& =\left[\sum_{i_{2}=1}^{m}\left[\left(\sum_{i_{1}, i_{2}}^{m} F\left(a_{i_{1} i_{2}}\right)\right) \cdot\left(\sum_{i_{2} i_{3}}^{m} G\left(a_{i_{2} i_{3}}\right)\right)\right]\right] \\
& =F_{m}\left(A_{1}\right) G_{m}\left(A_{2}\right),
\end{aligned}
$$

in $M_{m}(\mathbf{B}(\mathbf{H})) \otimes M_{n}(\mathbf{B}(\mathbf{H}))$.

Lemma 2 Let $F=\left[f_{i j}\right]: M_{n}(\mathbf{A}) \rightarrow M_{n}(\mathbf{B}(\mathbf{H}))$ and $G=\left[g_{i j}\right]: M_{n}(\mathbf{B}) \rightarrow M_{n}(\mathbf{B}(\mathbf{H}))$ be the linear operators. If the linear operators $F$ and $G$ are completely bounded, then the corresponding linear operator $\phi=F \odot G: M_{n}(\mathbf{A}) \odot M_{n}(\mathbf{B}) \rightarrow M_{n}(\mathbf{B}(\mathbf{H}))$ is completely bounded. In this case, we have $\|\phi\|_{c b}=\sup \left\{\left\|\phi_{n}\right\|\right\} \leq\|F\|_{c b} \cdot\|G\|_{c b}$.

Proof. First of all, we define completely bounded maps $f: \mathbf{E} \rightarrow \mathbf{B}(\mathbf{H}), g: \mathbf{F} \rightarrow \mathbf{B}(\mathbf{H})$ by

$$
\begin{aligned}
& f(e)=Y \pi(e) Y^{*} ; \\
& g(f)=Y S \rho(f) Z^{*} .
\end{aligned}
$$

For completely bounded operators, from Theorem 3 we can obtain

$$
\begin{aligned}
|\phi(e \otimes f)| & =\left\langle\left(\pi\left(e_{i j}\right)\right)\left(T_{i j}\right)\left(\rho\left(f_{i j}\right)\right) \xi, \eta\right\rangle=\left|(f \otimes g)_{n}(U)\right| \\
& =\left\langle F \odot G(V) D_{1}, D_{2}\right\rangle=|F \odot G(V)| .
\end{aligned}
$$


And more details can be found in Theorem 4. If $F$ and $G$ are completely bounded, we have

$$
\begin{aligned}
\|\phi\|_{c b} & =\sup \left\{\left\|\phi_{m}\left(D_{1}, D_{2}\right)\right\|\right\} \\
& =\sup \left\{\left\|(F \odot G)_{m}(V) D_{1}, D_{2}\right\|\right\} \\
& \leq \sup \left\{\left\|F_{m}\right\| \cdot\left\|G_{m}\right\|\right\}=\|F\|_{c b}\|G\|_{c b} .
\end{aligned}
$$

where $D_{1} \in M_{m}(\mathbf{A}), \quad D_{2} \in M_{m}(\mathbf{B}), \quad m \in N$. Hence $\phi$ is completely bounded with $\|\phi\|_{c b} \leq\|F\|_{c b}\|G\|_{c b}$ and this completes the proof.

Lemma 3 If $\phi \in(\mathbf{E} \otimes \mathbf{F})^{*}$ has norm one then there exist representations $\pi: \mathbf{A} \rightarrow \mathbf{B}(\mathbf{H}), \quad \rho: \mathbf{B} \rightarrow \mathbf{B}(\mathbf{H})$, a contraction $T: \mathbf{H} \rightarrow \mathbf{H}$, and unit vectors $\xi \in \mathbf{H}, \eta \in \mathbf{H}$ such that

$$
\phi(e \otimes f)=\langle\pi(e) T \rho(f) \xi, \eta\rangle
$$

Proof. By Proposition 2, $\phi$ is completely bounded, and so we may apply Theorem 3. The result now follows by setting $\xi=V_{1}(1), \quad \eta=V_{2}(1)$.

If $\phi: \mathbf{E} \rightarrow \mathbf{B}(\mathbf{H})$ and $\psi: \mathbf{F} \rightarrow \mathbf{B}(\mathbf{H})$ are bounded then there is a linear map $\phi \otimes \psi: \mathbf{E} \otimes_{h} \mathbf{F} \rightarrow \mathbf{B}(\mathbf{H})$ defined by

$$
\phi \otimes \psi\left(\sum_{i=1}^{r} e_{i} \otimes f_{i}\right)=\sum_{i=1}^{r} \phi\left(e_{i}\right) \otimes \psi\left(f_{i}\right)
$$

This map need not be bounded unless both $\phi$ and $\psi$ are completely bounded.

Consider the simplified form of multivariate switched linear systems described in (9), we give the result of the representation theorem as in the following.

Theorem 4 Consider the multivariate switched systems described in (9), the associated linear map $\phi: M_{n}(\mathbf{U}) \odot M_{n}(\mathbf{L}) \rightarrow M_{n}(\mathbf{Y}) \odot M_{n}(\mathbf{L})$ is completely bounded if and only if there are completely bounded linear functionals $f_{1}, f_{2}, \cdots, f_{n}$ and $g_{1}, g_{2}, \cdots, g_{n}$ on $\mathbf{E}$ such that $\phi=F \odot G$, where

$$
F=\left(\begin{array}{ccc}
f_{1} & \cdots & f_{n} \\
0 & \cdots & 0 \\
\vdots & \vdots & \vdots \\
0 & \cdots & 0
\end{array}\right), \quad G=\left(\begin{array}{ccc}
g_{1} & \cdots & g_{n} \\
0 & \cdots & 0 \\
\vdots & \vdots & \vdots \\
0 & \cdots & 0
\end{array}\right)
$$

The linear operators $F: M_{n}(\mathbf{U}) \rightarrow M_{n}(\mathbf{Y})$ and $G: M_{n}(\mathbf{U}) \rightarrow M_{n}(\mathbf{L})$ are completely bounded with $\|\phi\|_{c b} \leq\|F\|_{c b} \cdot\|G\|_{c b}$.

The proof can be found in the Appendix.

Remark 1 There can be no extension theorem for general bilinear maps. If $P$

$$
P=\left(\begin{array}{ccc}
p_{1} & \cdots & p_{n} \\
0 & \cdots & 0 \\
\vdots & \vdots & \vdots \\
0 & \cdots & 0
\end{array}\right)
$$

is another completely bounded linear operator from $M_{n}(\mathbf{U})$ into $M_{n}(\mathbf{Y})$ such that $\phi=P \odot G$, then there is a complex number $\lambda$ with $|\lambda|=1$ such that $F=\lambda P$.

Suppose that $P=\left(\begin{array}{cc}p_{1} & p_{2} \\ 0 & 0\end{array}\right): M_{n}(\mathbf{U}) \rightarrow M_{n}(\mathbf{Y})$ is another completely bounded linear operator such that $\phi=P \odot G$. Since $\|F\|_{c b}\|G\|_{c b} \geq\|\phi\|_{c b} \neq 0$, we may assume that $f_{1} \neq 0$ and $g_{1} \neq 0$. Then there is an element $x_{0}, y_{0} \in A$ such that $f_{1}^{*}\left(x_{0}\right) \neq 0, g_{1}^{*}\left(y_{0}\right) \neq 0$. Thus for all $x, y \in A$, we have

$$
\begin{aligned}
{\left[f_{i}(x) g_{j}(y)\right] } & =(F \odot G)(x, y)=\phi(x, y) \\
& =(P \odot G)(x, y)=\left[p_{i}(x) g_{j}(y)\right] .
\end{aligned}
$$


If we let $\lambda=\frac{p_{1}\left(x_{0}\right)}{f_{1}\left(x_{0}\right)}$, then we get $p_{i}(x)=\lambda f_{i}(x)$ for all $x \in A,(i=1,2)$, i.e. we get $P=\lambda F$. Since $\|\phi\|_{c b} \leq\|P\|_{c b}\|G\|_{c b}=\|F\|_{c b}\|G\|_{c b}=|\lambda|\|F\|_{c b}\|G\|_{c b}$, then $|\lambda|=1$.

Proposition 5 Consider multivariate switched linear systems described in (9) and (10) with the associated linear map: $\quad \phi: \mathbf{U} \otimes \mathbf{L} \rightarrow \mathbf{B}(\mathbf{H})$, if the open or closed-loop switched linear systems is globally stable or globally asymptotically stable, then there exist representations $\pi: \mathbf{U} \rightarrow \mathbf{B}(\mathbf{Y}), \rho: \mathbf{L} \rightarrow \mathbf{B}(\mathbf{L})$, isometries: $V_{1}: \mathbf{H} \rightarrow \mathbf{Y}$ and $V_{2}: \mathbf{H} \rightarrow \mathbf{L}$, and a contraction $T: \mathbf{Y} \rightarrow \mathbf{L}$ such that on elementary tensors

$$
\phi(u \otimes l)=V_{1}^{*} \pi(u) T \rho(l) V_{2}
$$

where $u \in \mathbf{U}, l \in \mathbf{L}$.

Proof. Applying Theorem 3, the result is followed.

From the expressions (11) in Theorem 3 and (23) in Proposition 5, it is shown that the bilinear systems and switched linear systems are the same with the linear systems representation form. However, from (7) of the switched linear systems description in previous section, it can be found that systems outputs determine the logical switching signals described in (10), which shows the contraction $T$ in Proposition 5 is determined in the operator itself (or switched linear systems itself). Furthermore, the logical functions outputs values are the logical values 0 or 1 , so the logical functions outputs values are determined for the entire dynamical systems, of course, for the active subsystem, the logical value is 1 . More details will be shown in the proof of Theorem 7.

Theorem 6 [22] Let $\mathbf{H}$ be a fixed infinite dimensional separable Hilbert space. The norm in $M_{n}\left(\mathbf{E} \otimes_{h} \mathbf{F}\right)$ satisfies

$$
\|U\|_{n}=\sup \left\{(\phi \otimes \psi)_{n}(U)\right\}
$$

where the supremum is taken over all complete contractions $\phi: \mathbf{E} \rightarrow \mathbf{B}(\mathbf{H}), \psi: \mathbf{F} \rightarrow \mathbf{B}(\mathbf{H})$.

Lemma 4 Consider multivariate switched linear systems described in (9) and (10). Let $F=\left[f_{i j}\right]: M_{n}(\mathbf{U}) \rightarrow M_{n}(\mathbf{Y})$ be the linear functional and $G=\left[g_{i j}\right]: M_{n}(\mathbf{L}) \rightarrow M_{n}(\mathbf{L})$ be the logical functional. Then, if the linear functional $F$ is completely bounded and the logical functional $G$ is nonzero projection with $\|G\|_{c b}=1$, the corresponding tensor-product representation $\phi=F \odot G: M_{n}(\mathbf{U}) \odot M_{n}(\mathbf{L}) \rightarrow M_{n}(\mathbf{Y}) \odot M_{n}(\mathbf{L})$ is completely bounded. In this case, we have $\|\phi\|_{c b}=\sup \left\{\left\|\phi_{n}\right\|\right\}=\inf \left\{\|F\|_{c b}\|G\|_{c b}\right\}=\inf \left\{\|F\|_{c b}\right\}$.

Proof. Applying Theorem 6, the result is followed.

A necessary and sufficient conditions for tensor-product representation of multivariate switched linear systems can now be presented, under the assumption that the open or closed-loop switched linear systems is globally stable or globally asymptotically stable.

Theorem 7 Consider multivariate switched linear systems described in (9) and (10), the tensor-product representation $\phi: M_{n}(\mathbf{U}) \odot M_{n}(\mathbf{L}) \rightarrow M_{n}(\mathbf{Y}) \odot M_{n}(\mathbf{L})$ is globally stable or globally asymptotically stable if and only if there are completely bounded linear functionals $f_{1}, f_{2}, \cdots, f_{n}$ on $M_{n}(\mathbf{U})$ and the logical switching functionals $g_{1}, g_{2}, \cdots, g_{n}$ on $M_{n}(\mathbf{L})$ such that $\phi=F \odot G$, where

$$
F=\left(\begin{array}{ccc}
f_{1} & \cdots & f_{n} \\
0 & \cdots & 0 \\
\vdots & \vdots & \vdots \\
0 & \cdots & 0
\end{array}\right)
$$

the linear function $F: M_{n}(\mathbf{U}) \rightarrow M_{n}(\mathbf{Y})$ is completely bounded, and

$$
G=\left(\begin{array}{ccc}
g_{1} & \cdots & g_{n} \\
0 & \cdots & 0 \\
\vdots & \vdots & \vdots \\
0 & \cdots & 0
\end{array}\right)
$$

the logical function $G: M_{n}(\mathbf{L}) \rightarrow M_{n}(\mathbf{L}),\|G\|_{c b}=1$ is nonzero logical value projection with $\|\phi\|_{c b}=\inf \left\{\|F\|_{c b}\right\}$.

The proof can be found in the Appendix. 
It is noted that the $F$ in Theorem 4 is defined to be taken over all representations for the linear operators, but the $F$ in Theorem 7 is defined to be taken over all representations of the subsystems operators for multivariate switched linear systems. This difference naturally lead to the different tensor-product representations for the general bilinear systems and the multivariate switched linear systems, and the $n$ in $M_{n}$ can be interpreted as the integer of the all finite switched subsystems of multivariate switched linear systems. More details can be found in the Examples 1 and 2.

Remark 2 Theorem 7 mainly considers the logic switching space, which exactly describes the tensor-product algebra representation for multivariate switched linear systems, which can extend into the scenario of the timevarying disturbance dynamics. And Theorem 4 just considers the tensor-product algebra representation structure for general bilinear systems operators, which can also extend into more general class of multivariate nonlinear systems in Theorem 10 in this paper. In addition, Theorem 4 shows the tensor-product representation structure for general bilinear operator and Theorem 7 shows the tensor-product representation structure for multivariate switched linear systems in the framework of bilinear algebra. Furthermore, multivariate switched linear systems essentially can be interpreted as the special class of general bilinear operator with logical linear operator substituting the general linear operators.

Next, we give an example (including operator description and unfolding form) of tensor-product representation for multivariate switched linear systems from the perspective of the structured systems models subject to the explicitly bounded disturbance which guarantees the entire systems are completely bounded.

Example 1 Let the $k$-th subsystem transfer function $\mathbf{G}_{\sigma_{k}}$ be given as

$$
\mathbf{G}_{\sigma_{k}}=\left[\begin{array}{cccc}
\mathbf{G}_{\sigma_{k}}^{1,1} & \mathbf{G}_{\sigma_{k}}^{1,2} & \cdots & \mathbf{G}_{\sigma_{k}}^{1, n} \\
\mathbf{G}_{\sigma_{k}}^{2,1} & \mathbf{G}_{\sigma_{k}}^{2,2} & \cdots & \mathbf{G}_{\sigma_{k}}^{2, n} \\
\vdots & \vdots & \ddots & \vdots \\
\mathbf{G}_{\sigma_{k}}^{m, 1} & \mathbf{G}_{\sigma_{k}}^{m, 2} & \cdots & \mathbf{G}_{\sigma_{k}}^{m, n}
\end{array}\right]
$$

For the dynamical systems, the tensor-product representation for the operator systems can be obtained as

$$
F \otimes G_{k}=\left[\lambda_{1} \mathbf{G}_{\sigma_{1}}+\lambda_{2} \mathbf{G}_{\sigma_{2}}+\cdots+\lambda_{k} \mathbf{G}_{\sigma_{k}}+\cdots+\lambda_{K} \mathbf{G}_{\sigma_{K}}+\mathbf{H}\right] \otimes\left[\begin{array}{cccc}
\lambda_{i} & 0 & \cdots & 0 \\
0 & 0 & \cdots & 0 \\
\vdots & \vdots & \ddots & \vdots \\
0 & 0 & \cdots & 0
\end{array}\right]
$$

where $\lambda_{k} \cdot \lambda_{i}=1, i=k$, or $\lambda_{k} \cdot \lambda_{i}=0, i \neq k$, also $\lambda_{i} \mathbf{H}=\mathbf{H}, i, k \in\{1,2, \cdots, K\}$. The input space is $u_{\lambda_{k}}$ and the logical switching space is $\left[\begin{array}{lllll}0 & \cdots & \lambda_{k} & \cdots & 0\end{array}\right]$. For the entire switched linear systems, the tensor-product representation for the operator systems is given as

$$
F \odot G=\left[\begin{array}{llll}
f_{1} & f_{2} & \cdots & f_{K}
\end{array}\right] \odot\left[\begin{array}{cccc}
\lambda_{1} & 0 & \cdots & 0 \\
0 & \lambda_{2} & \cdots & 0 \\
\vdots & \vdots & \ddots & \vdots \\
0 & 0 & \cdots & \lambda_{K}
\end{array}\right]
$$

where $f_{1}=f_{2}=\cdots=f_{K}=f, f=\lambda_{1} \mathbf{G}_{\sigma_{1}}+\lambda_{2} \mathbf{G}_{\sigma_{2}}+\cdots+\lambda_{k} \mathbf{G}_{\sigma_{k}}+\cdots+\lambda_{K} \mathbf{G}_{\sigma_{K}}+\mathbf{H}, \lambda_{k} \cdot \lambda_{i}=1, i=k$, or $\lambda_{k} \cdot \lambda_{i}=0$, $i \neq k$, also $\lambda_{i} \mathbf{H}=\mathbf{H}, \quad i, k \in\{1,2, \cdots, K\}$. The input space is $u_{\lambda_{k}}$ and the logical switching sequence is $L=\left[\begin{array}{lllll}\lambda_{1} & \cdots & \lambda_{k} & \cdots & \lambda_{K}\end{array}\right]$. Here, we assume that each subsystem just performs only once for the entire dynamical systems. Consequently, the tensor-product representation for the input space can be obtained as $\mathbf{U} \odot \mathbf{L}$ and the corresponding output space is $\mathbf{Y} \odot \mathbf{L}$.

Intuitively, the systems description can be interpreted as considering a linear combination of the linear operators of the original switched linear system that evolves in an asymptotically stable manner in a subspace. The tensor-product representation algebra system evolves in a higher dimensional space to which the original system can be projected under the stability condition of the entire multivariate switched linear systems. From Example 1, we can see that the multivariate switched dynamical systems can be expressed in the form of tensor-product extending the matrix description of multivariate linear systems.

Remark 3 For the entire switched linear systems, the tensor-product representation $\phi$ can be interpreted as 
the linear systems form expressed as $F \odot G$. Therefore, under the stability conditions of the systems, the control systems performance measure is attainable. It is shown that the multivariate switched linear systems can be transformed into the linear systems expression form via tensor-product representation technique, which is convenient to develop the controller performance assessment methods for such class of nonlinear systems.

Proposition 8 Let $V: \mathbf{E}_{1} \times \mathbf{E}_{2} \times \cdots \times \mathbf{E}_{n} \rightarrow \mathbf{B}(\mathbf{H})$ be multi-linear maps and let $\phi$ be its associated linear map, then the $\|\phi\|_{c b}=\|V\|_{c b}$.

Proof. The proof follows that of Proposition 2.

Theorem 9 [22] Let $\left\{\mathbf{E}_{i}\right\}_{i=1}^{n}$ be subspaces of $C_{*}$-algebras $\left\{\mathbf{A}_{i}\right\}_{i=1}^{n}$, and let $V: \mathbf{E}_{1} \times \cdots \times \mathbf{E}_{n} \rightarrow \mathbf{B}(\mathbf{H})$ be a completely bounded multilinear map. Then there exist Hilbert spaces $\mathbf{H}_{i}, 1 \leq i \leq n$, representations $\pi_{i}$, $\mathbf{A}_{i} \rightarrow \mathbf{B}\left(\mathbf{H}_{i}\right), \quad 1 \leq i \leq n$, isometries $V_{i}: \mathbf{H} \rightarrow \mathbf{H}_{i}, i=1, n$ and contractions $T_{i}: \mathbf{H}_{i+1} \rightarrow \mathbf{H}_{i}, 1 \leq i \leq n-1$, such that,

$$
V\left(e_{1}, \cdots, e_{n}\right)=V_{1}^{*} \pi_{1}\left(e_{1}\right) T_{1} \pi_{2}\left(e_{2}\right) T_{2} \cdots T_{n-1} \pi_{n}\left(e_{n}\right) V_{n}
$$

The construction of efficient representations to multivariate functions and related operators plays a crucial role in the numerical analysis of higher dimensional problems arising in a wide range of modern applications. We generalize our results in Theorem 4 to the k-linear operators.

Theorem 10 Let $\phi: M_{n}\left(\mathbf{U}^{k}\right) \rightarrow M_{n}(\mathbf{B}(\mathbf{H}))$, Then $\phi$ is completely bounded if and only if there are completely bounded linear functionals $f_{1}^{i}, f_{2}^{i}, \cdots, f_{n}^{i}$ with $i=1,2, \cdots, k$ on $\mathbf{U}$ such that $\phi=F_{1} \odot F_{2} \odot \cdots \odot F_{k}$, where

$$
F_{i}=\left(\begin{array}{ccc}
f_{1}^{i} & \cdots & f_{n}^{i} \\
0 & \cdots & 0 \\
\vdots & \ddots & \vdots \\
0 & \cdots & 0
\end{array}\right)
$$

The $i$-th linear operator $F_{i}$ is completely bounded with $\|\phi\|_{c b} \leq\left\|F_{1}\right\|_{c b}\left\|F_{2}\right\|_{c b} \cdots\left\|F_{k}\right\|_{c b}$.

The proof can be found in the Appendix.

Remark 4 It is difficult to describe the multivariate nonlinear systems in practical industrial applications. The analytic methods developed in [31] [32] for tensor-product approximations to multidimensional integral operators play an important role in the nonlinear control systems performance assessment. For the case of collocation schemes it focuses on the construction of tensor decompositions which are exponentially convergent in the separation rank. Separable approximation of functions and tensors can be derived by using a corresponding separable expansion of the generating function, including a separable approximation of multivariate functions, and the tensor-product approximation of some analytic matrix-valued functions. In practical applications, the general error estimation should mainly consider in the tensor-product approximations for multivariate switched linear systems. In the near future, we will apply analytically-based representation methods, which are efficient for a special class of function-related operators/tensors. The key observation is that there is a natural duality between separable approximation of the multivariate generating function and the tensor-product decomposition of the related multidimensional array which is suitable for geometric quantization for control systems performance assessment.

Corollary 2 A $k$-linear operator from $\mathbf{A}^{k}$ into $\mathbf{B}(\mathbf{H})$ is representable if and only if it is completely bounded; when $\phi$ is completely bounded, $\|\phi\|_{c b}=\|\phi\|_{\text {rep }}$, and the representable norm $\|\phi\|_{\text {rep }}$ is attained.

Proof. From the Theorem 9 and Theorem 10, the results is followed.

Next, we extend the multivariate switched linear systems to the case of time varying disturbance scenario. We also assume that disturbance dynamics are piecewise linear time varying. This type of time varying disturbances are reflected as different output trajectories in different subprocess, each subprocess having the same linear time invariant disturbance models. In other words, it is assumed that the disturbance models are the same within each subprocess, and it is required that the switching sequence of disturbance models is also the same within each subprocess. We will give the tensor-product algebraic representation from the point of view of operator theory in the following.

Theorem 11 Consider multivariate switched linear systems described in (9) and (10) with time varying disturbance dynamics, the tensor-product representation $\phi: M_{n}(\mathbf{U}) \odot M_{n}(\mathbf{L}) \odot M_{d}(\mathbf{D}) \rightarrow M_{n}(\mathbf{Y}) \odot M_{n}(\mathbf{L}) \odot M_{d}(\mathbf{D})$ is globally stable or globally asymptotically stable if and only if there are completely bounded linear functionals 
$f_{1}, f_{2}, \cdots, f_{n}$ on $M_{n}(\mathbf{U})$, the logical switching functionals $g_{1}, g_{2}, \cdots, g_{n}$ on $M_{n}(\mathbf{L})$ and the disturbance logic switching functionals $h_{1}, h_{2}, \cdots, h_{d}$ on $M_{d}(\mathbf{D})$; the linear function $F: M_{n}(\mathbf{U}) \rightarrow M_{n}(\mathbf{Y})$ is completely bounded, the logical switching function $G: M_{n}(\mathbf{L}) \rightarrow M_{n}(\mathbf{L})$ with $\|G\|_{c b}=1$ and the disturbance logic switching function $H: M_{d}(\mathbf{D}) \rightarrow M_{d}(\mathbf{D})$ with $\|H\|_{c b}^{n}=1$ are nonzero value projections, then $\phi=F \odot G \odot H$ with $\|\phi\|_{c b}=\inf \left\{\|F\|_{c b}\|G\|_{c b}\|H\|_{c b}\right\}=\inf \left\{\|F\|_{c b}\right\}$.

From the Lemma 4 and Theorem 9, the results is followed.

Now, we will give an example of tensor-product representation for multivariate switched linear systems with time-varying disturbance dynamics. The representation results is constructive for practical industrial process systems modeling framework.

Example 2 A possible tensor expression of system (6) can be described for all the K MIMO subsystems in the tensor-product representation form. And, the j-th disturbance transfer function $\mathbf{H}_{\delta_{j}}$ is given as

$$
\mathbf{H}_{\delta_{j}}=\left[\begin{array}{cccc}
\mathbf{H}_{\delta_{j}}^{1,1} & \mathbf{H}_{\delta_{j}}^{1,2} & \cdots & \mathbf{H}_{\delta_{j}}^{1, q} \\
\mathbf{H}_{\delta_{j}}^{2,1} & \mathbf{H}_{\delta_{j}}^{2,2} & \cdots & \mathbf{H}_{\delta_{j}}^{2, q} \\
\vdots & \vdots & \ddots & \vdots \\
\mathbf{H}_{\delta_{j}}^{m, 1} & \mathbf{H}_{\delta_{j}}^{m, 2} & \cdots & \mathbf{H}_{\delta_{j}}^{m, q}
\end{array}\right]
$$

For the dynamical systems, the tensor-product representation for the operator systems can be obtained as

$$
\begin{aligned}
F \otimes G_{k} \otimes H_{j}= & {\left[\lambda_{1} \mathbf{G}_{\sigma_{1}}+\lambda_{2} \mathbf{G}_{\sigma_{2}}+\cdots+\lambda_{k} \mathbf{G}_{\sigma_{k}}+\cdots+\lambda_{K} \mathbf{G}_{\sigma_{K}}+\mu_{1} \mathbf{H}_{\sigma_{1}}+\mu_{2} \mathbf{H}_{\sigma_{2}}+\cdots+\mu_{j} \mathbf{H}_{\sigma_{j}}+\cdots+\mu_{J} \mathbf{H}_{\sigma_{J}}\right] } \\
\otimes & {\left[\begin{array}{cccc}
\lambda_{i} & 0 & \cdots & 0 \\
0 & 0 & \cdots & 0 \\
\vdots & \vdots & \ddots & \vdots \\
0 & 0 & \cdots & 0
\end{array}\right] \otimes\left[\begin{array}{cccc}
\mu_{1} & 0 & \cdots & 0 \\
0 & 0 & \cdots & 0 \\
\vdots & \vdots & \ddots & \vdots \\
0 & 0 & \cdots & 0
\end{array}\right], }
\end{aligned}
$$

where $\lambda_{k} \cdot \lambda_{i}=1, k=i$, or $\lambda_{k} \cdot \lambda_{i}=0, k \neq i, i, k \in\{1,2, \cdots, K\} ; \mu_{j} \cdot \mu_{l}=1, j=l$, or $\mu_{j} \cdot \mu_{l}=0, j \neq l$, $j, l \in\{1,2, \cdots, J\}$; also, $\lambda_{k} \mathbf{G}_{\sigma_{k}} \mu_{j}=\lambda_{1} \mathbf{G}_{\sigma_{k}}, \mu_{j} \mathbf{H}_{\sigma_{j}} \lambda_{k}=\mu_{1} \mathbf{H}_{\sigma_{j}}, k \in\{1,2, \cdots, K\}, j \in\{1,2, \cdots, J\}$. The input space is $u_{\lambda_{k}}$, the logical switching space is $\left[\begin{array}{llllll}0 & \cdots & \lambda_{k} & \cdots & 0\end{array}\right]$, and the disturbance logic switching space is $\left[\begin{array}{lllll}0 & \cdots & \mu_{j} & \cdots & 0\end{array}\right]$.

For the entire switched systems, the tensor-product representation for the operator systems is given as

$$
\begin{aligned}
& F \odot G \odot H=\left[\begin{array}{llll}
f_{1} & f_{2} & \cdots & f_{K}
\end{array}\right] \odot\left[\begin{array}{cccc}
\lambda_{1} & 0 & \cdots & 0 \\
0 & \lambda_{2} & \cdots & 0 \\
\vdots & \vdots & \ddots & \vdots \\
0 & 0 & \cdots & \lambda_{K}
\end{array}\right]
\end{aligned}
$$

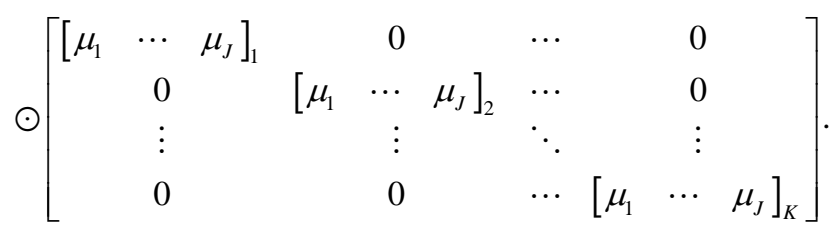

where $f_{1}=f_{2}=\cdots=f_{K}=f$, and

$f=\lambda_{1} \mathbf{G}_{\sigma_{1}}+\lambda_{2} \mathbf{G}_{\sigma_{2}}+\cdots+\lambda_{k} \mathbf{G}_{\sigma_{k}}+\cdots+\lambda_{K} \mathbf{G}_{\sigma_{K}}+\mu_{1} \mathbf{H}_{\sigma_{1}}+\mu_{2} \mathbf{H}_{\sigma_{2}}+\cdots+\mu_{j} \mathbf{H}_{\sigma_{j}}+\cdots+\mu_{J} \mathbf{H}_{\sigma_{J}}, \lambda_{k} \cdot \lambda_{i}=1, \quad k=i$, or $\lambda_{k} \cdot \lambda_{i}=0, \quad k \neq i, \quad i, k \in\{1,2, \cdots, K\} ; \quad \mu_{j} \cdot \mu_{l}=1, \quad j=l$, or $\mu_{j} \cdot \mu_{l}=0, j \neq l, j, l \in\{1,2, \cdots, J\}$; also, $\lambda_{k} \mathbf{G}_{\sigma_{k}} \mu_{j}=\lambda_{1} \mathbf{G}_{\sigma_{k}}, \quad \mu_{j} \mathbf{H}_{\sigma_{j}} \lambda_{k}=\mu_{1} \mathbf{H}_{\sigma_{j}}, k \in\{1,2, \cdots, K\}, \quad j \in\{1,2, \cdots, J\}$. The k-subsystem input under j-disturbance mode is $u_{\lambda_{k} \mu_{j}}$, the subsystems logical switching sequence is $L=\left[\begin{array}{lllll}\lambda_{1} & \cdots & \lambda_{k} & \cdots & \lambda_{K}\end{array}\right]$ and the disturbance switching sequence $D=\left[\begin{array}{lllll}\mu_{1} & \cdots & \mu_{j} & \cdots & \mu_{J}\end{array}\right]$. Here, we assume that each subsystem just performs only once for the entire dynamical systems. Consequently, the tensor-product representation for the input space can be obtained as $\mathbf{U} \odot \mathbf{L} \odot \mathbf{D}$ and the corresponding output space is $\mathbf{Y} \odot \mathbf{L} \odot \mathbf{D}$. The tensor space expression form for multivariate switched linear systems with time-varying disturbance dynamics is given as 


$$
\mathcal{Y}=\mathcal{G} u_{t}+\mathcal{H} a_{t}
$$

where $\mathcal{Y}=\left[\mathbf{Y}_{J, 1}, \mathbf{Y}_{J, 2}, \cdots, \mathbf{Y}_{J, K}\right] \in \mathbf{R}^{m \times J \times K}$ is the three-order tensor, and $\mathcal{G} \in \mathbf{R}^{m \times p \times J \times K}$ and $\mathcal{H} \in \mathbf{R}^{m \times q \times J \times K}$ are the four-order tensors.

\section{Conclusion}

A new tensor-product representation for describing the dynamic behavior of switched linear systems has been presented. The tensor-product representation makes it easy to see the relationship between abstractly algebraic expressions that propagate spatial quantities from subsystem-to-subsystem. Abstract dynamic equations arising from a hybrid systems analysis, can be reinterpreted as equivalent operator-formulated equations. This algebra expression can model the behavior of the switched linear systems by the use of a tensor-product representation, which shows that the algebraic structure can simplify switched linear systems expression. The proposed technique is well suited for real and complex systems, and is capable of providing the tensor-product representation of the class of stable nonlinear system. Furthermore, the interpretation of expressions within the tensor-product representation framework can be enhanced conceptual and physical understanding of switched linear systems, or more general of multivariate nonlinear systems, dynamic behavior expression.

\section{References}

[1] Goebel, R., Sanfelice, R.G. and Teel, A.R. (2009) Hybrid Dynamical Systems. IEEE Control Systems Magazine, 29, 28-93. http://dx.doi.org/10.1109/MCS.2008.931718

[2] Liberzon, D. and Morse, A.S. (1999) Basic Problems in Stability and Design of Switched Systems. IEEE Control Systems Magazine, 19, 59-70. http://dx.doi.org/10.1109/37.793443

[3] Decarlo, R.A., Branicky, M.S., Pettersson, S. and Lennartson, B. (2000) Perspectives and Results on the Stability and Stabilization of Hybrid Systems. Proceedings of the IEEE, 88, 1069-1082. http://dx.doi.org/10.1109/5.871309

[4] Hu, L.S., Cao, Y.Y. and Shao, H.H. (2002) Constrained Robust Sampled-Data Control for Nonlinear Uncertain Systems. International Journal of Robust and Nonlinear Control, 12, 447-464. http://dx.doi.org/10.1002/rnc.632

[5] Hu, L.S., Lam, J., Cao, Y.Y. and Shao, H.H. (2003) LMI Approach to Robust $H_{2}$ Sampled-Data Control for Linear Uncertain Systems. IEEE Transactions on Systems Man and Cybernetics Part B, 33, 149-155. http://dx.doi.org/10.1109/TSMCB.2003.808181

[6] Hu, L.S., Shi, P. and Huang, B. (2005) $H_{\infty}$ Control for Sampled-Data Linear Systems with Two Markovian Processes. Optimal Control Applications \& Methods, 26, 291-306. http://dx.doi.org/10.1002/oca.761

[7] Hu, L.S., Shi, P. and Frank, P.M. (2006) Robust Sampled-Data Control for Markovian Jump Linear Systems. Automatica, 42, 2025-2030. http://dx.doi.org/10.1016/j.automatica.2006.05.029

[8] Hu, L.S., Bai, T., Shi, P. and Wu, Z.M. (2007) Sampled Data Control of Networked Linear Control Systems. Automatica, 43, 903-911. http://dx.doi.org/10.1016/j.automatica.2006.11.015

[9] Shi, P. (1998) Filtering on Sampled-Data Systems with Parametric Uncertainty. IEEE Transactions on Automatic Control, 43, 1022-1027. http://dx.doi.org/10.1109/9.701119

[10] Hu, L.S., Shi, P. and Huang, B. (2006) Stochastic Stability and Robust Control for Sampled-Data Systems with Markovian Jump Parameters. Journal of Mathematical Analysis and Applications, 313, 504-517. http://dx.doi.org/10.1016/j.jmaa.2005.08.019

[11] Shi, P., Boukas, E.K. and Agarwal, R.K. (1999) Control of Markovian Jump Discrete-Time Systems with Norm Bounded Uncertainty and Unknown Delay. IEEE Transactions on Automatic Control, 44, 2139-2144. http://dx.doi.org/10.1109/9.802932

[12] Shi, P., Xia, Y., Liu, G.P. and Rees, D. (2006) On Designing of Sliding-Mode Control for Stochastic Jump Systems. IEEE Transactions on Automatic Control, 51, 97-103. http://dx.doi.org/10.1109/TAC.2005.861716

[13] Skafidas, E., Evans, R.J., Savkin, A.V. and Petersen, I.R. (1999) Stability Results for Switched Controller Systems. Automatica, 35, 553-564. http://dx.doi.org/10.1016/S0005-1098(98)00167-8

[14] Narendra, K.S., Driollet, O.A., Feiler, M. and George, K. (2003) Adaptive Control Using Multiple Models, Switching and Tuning. International Journal of Adaptive Control and Signal Processing, 17, 87-102. http://dx.doi.org/10.1002/acs.740

[15] Lin, H. and Antsaklis, P.J. (2007) Switching Stabilizability for Continuous-Time Uncertain Switched Linear Systems. IEEE Transactions on Automatic Control, 52, 633-646. http://dx.doi.org/10.1109/TAC.2007.894515 
[16] Lin, H. and Antsaklis, P.J. (2009) Stability and Stabilizability of Switched Linear Systems: A Survey of Recent Results. IEEE Transactions on Automatic Control, 54, 308-322. http://dx.doi.org/10.1109/TAC.2008.2012009

[17] Sun, Z. and Ge, S.S. (2005) Analysis and Synthesis of Switched Linear Control Systems. Automatica, 41, $181-195$. http://dx.doi.org/10.1016/j.automatica.2004.09.015

[18] Zhai, G., Lin, H. and Antsaklis, P. (2003) Quadratic Stabilizability of Switched Linear Systems with Polytopic Uncertainties. International Journal of Control, 76, 747-753. http://dx.doi.org/10.1080/0020717031000114968

[19] Athans, M. (1987) Command and Control (C2) Theory: A Challenge to Control Science. IEEE Transactions on Automatic Control, 32, 286-293. http://dx.doi.org/10.1109/TAC.1987.1104607

[20] Zhao, X., Zhang, L., Shi, P. and Liu, M. (2012) Stability of Switched Positive Linear Systems with Average Dwell Time Switching. Automatica, 48, 1132-1137. http://dx.doi.org/10.1016/j.automatica.2012.03.008

[21] Effros, E.G. and Ruan, Z.J. (1988) Representations of Operator Bimodules and Their Applications. Journal of Operator Theory, 19, 137-157.

[22] Paulsen, V.I. and Smith, R.R. (1987) Multilinear Maps and Tensor Norms on Operator Systems. Journal of Functional Analysis, 73, 258-276. http://dx.doi.org/10.1016/0022-1236(87)90068-1

[23] Ruan, Z.J. (1988) Subspaces of $C^{*}$-Algebras. Journal of Functional Analysis, 76, 217-230. http://dx.doi.org/10.1016/0022-1236(88)90057-2

[24] Paulsen, V.I. (1984) Every Completely Polynomially Bounded Operator Is Similar to a Contraction. Journal of Functional Analysis, 55, 1-17. http://dx.doi.org/10.1016/0022-1236(84)90014-4

[25] Effros, E.G. and Ruan, Z.J. (1988) On Matricially Normed Spaces. Pacific Journal of Mathematics, 132, $243-264$. http://dx.doi.org/10.2140/pjm.1988.132.243

[26] Paulsen, V.I. (1986) Completely Bounded Maps and Dilations. Pitman Research Notes in Mathematics, Longman, London.

[27] Christensen, E. and Sinclair, A.M. (1987) Representations of Completely Bounded Multilinear Operators. Journal of Functional Analysis, 72, 151-181. http://dx.doi.org/10.1016/0022-1236(87)90084-X

[28] Christensen, E. and Sinclair, A.M. (1989) A Survey of Completely Bounded Operators. Bulletin London Mathematical Society, 21, 417-448. http://dx.doi.org/10.1112/blms/21.5.417

[29] Choi, M.D. and Effros, E.G. (1977) Injectivity and Operator Spaces. Journal of Functional Analysis, 24, 159-209. http://dx.doi.org/10.1016/0022-1236(77)90052-0

[30] Effros, E.G. (1987) Advances in Quantized Functional Analysis. Proceedings of the International Congress of Mathematicians, Vol. 1, 2 (Berkeley, Calif., 1986), 906-916, Amer. Math. Soc., Providence, RI.

[31] Tyrtyshnikov, E. (2004) Kronecker-Product Approximations for Some Function-Related Matrices. Linear Algebra and Its Applications, 379, 423-437. http://dx.doi.org/10.1016/j.laa.2003.08.013

[32] Hackbusch, W. and Khoromskij, B.N. (2007) Tensor-Product Approximation to Operators and Functions in High Dimensions. Journal of Complexity, 23, 697-714. http://dx.doi.org/10.1016/j.jco.2007.03.007 


\section{Appendix}

\section{A.1. Proof of Theorem 4}

Proof. The necessity and sufficiency proofs are in the following.

Necessity of proof: Let $\phi: M_{n}(\mathbf{U}) \odot M_{n}(\mathbf{L}) \rightarrow M_{n}(\mathbf{B}(\mathbf{H})), \phi \neq 0$ be completely bounded. By applying Theorem 3 and Lemma 3, there are representations $\pi: \mathbf{A} \rightarrow \mathbf{B}\left(\mathbf{H}_{1}\right), \rho: \mathbf{B} \rightarrow \mathbf{B}\left(\mathbf{H}_{2}\right)$, unit vectors $\xi=\left(\xi_{1}, \cdots, \xi_{n}\right) \in \mathbf{H}_{2} \oplus \cdots \oplus \mathbf{H}_{2}, \quad \eta=\left(\eta_{1}, \cdots, \eta_{n}\right) \in \mathbf{H}_{1} \oplus \cdots \oplus \mathbf{H}_{1}$, and $T=\left(T_{i j}\right): \mathbf{H}_{2} \oplus \cdots \oplus \mathbf{H}_{2} \rightarrow \mathbf{H}_{1} \oplus \cdots \oplus \mathbf{H}_{1}$, such that

$$
\phi\left(\left(u_{i j}\right) \otimes\left(l_{i j}\right)\right)=\left\langle\left(\pi\left(u_{i j}\right)\right)\left(T_{i j}\right)\left(\rho\left(l_{i j}\right)\right) \xi, \eta\right\rangle
$$

for $\left(u_{i j}\right) \in M_{n}(\mathbf{U}), \quad\left(l_{i j}\right) \in M_{n}(\mathbf{L})$. Let $P$ and $Q$ be the projections onto the closures of $\left[\operatorname{span}\left\{\rho(l) \xi_{i}\right\}\right]$ and $\left[\operatorname{span}\left\{\pi\left(u^{*}\right) \eta_{i}\right\}\right]$. Then

$$
\phi\left(\left(u_{i j}\right) \otimes\left(l_{i j}\right)\right)=\left\langle\left(\pi\left(u_{i j}\right)\right)\left(Q T_{i j} P\right)\left(\rho\left(l_{i j}\right)\right) \xi, \eta\right\rangle
$$

Note that if $X$ is any unitary matrix in $M_{n}$, then

$$
\left(u_{i j}\right) X \otimes X^{*}\left(l_{i j}\right)-\left(u_{i j}\right) \otimes\left(l_{i j}\right) \in \operatorname{ker} \wedge
$$

Such elements are annihilated by $\phi$, and this leads to

$$
X\left(Q T_{i j} P\right) X^{*}=\left(Q T_{i j} P\right)
$$

for all unitaries $X$. This forces the matrix $\left(Q T_{i j} P\right)$ to have the diagonal form $\left(\begin{array}{lll}S & & \\ & \ddots & \\ & & S\end{array}\right)$, where $S: H_{2} \rightarrow H_{1}$, $\|S\| \leq 1$.

The element $V \in M_{n}(\mathbf{U}) \otimes_{h} M_{n}(\mathbf{L})$ can be represented using only a finite number of vectors $u_{1}, \cdots, u_{s} \in \mathbf{U}$ and $l_{1}, \cdots, l_{k} \in \mathbf{L}$. Define $\mathbf{J}_{1}=\operatorname{span}\left[\eta_{j}, \pi\left(u_{i}^{*}\right) \eta_{j}\right]$ and $\mathbf{J}_{2}=\operatorname{span}\left[\xi_{j}, \rho\left(l_{i}\right) \xi_{j}\right]$. Choose a separable Hilbert space $\mathbf{H}$ and norm one operators $Y: \mathbf{H}_{1} \rightarrow \mathbf{H}, \quad Z: \mathbf{H}_{2} \rightarrow \mathbf{H}$ which are isometric on $\mathbf{J}_{1}$ and $\mathbf{J}_{2}$ and define completely bounded maps $f: \mathbf{U} \rightarrow \mathbf{B}(\mathbf{H}), g: \mathbf{L} \rightarrow \mathbf{B}(\mathbf{H})$ by

$$
\begin{aligned}
& f(u)=Y \pi(u) Y^{*} ; \\
& g(l)=Y S \rho(l) Z^{*} .
\end{aligned}
$$

With these definitions it is easy to check that

$$
\phi(V)=\left\langle(F \odot G)(V) D_{1}, D_{2}\right\rangle
$$

where $D_{1}=\left(\begin{array}{c}Z \xi_{1} \\ \vdots \\ Z \xi_{n}\end{array}\right), \quad D_{2}=\left(\begin{array}{c}Y \eta_{1} \\ \vdots \\ Y \eta_{n}\end{array}\right)$, this implies that $\phi=F \odot G$. It follows from Lemma 2 that

$F: M_{n}(\mathbf{U}) \rightarrow M_{n}(\mathbf{Y})$ and $G: M_{n}(\mathbf{L}) \rightarrow M_{n}(\mathbf{L})$ are completely bounded with $\|F\|_{c b}\|G\|_{c b} \geq\|\phi\|_{c b}$. This completes the necessity proof.

Sufficiency of proof: It follows, from Lemma 2, that $\phi$ is a completely bounded linear operator from $M_{n}(\mathbf{U}) \odot M_{n}(\mathbf{L})$ into $M_{n}(\mathbf{B}(\mathbf{H}))$ with $\|\phi\|_{c b} \leq\|F\|_{c b}\|G\|_{c b}$. It suffices to show that the associated bilinear operator $V$ is completely bounded from $M_{n}(\mathbf{A}) \times M_{n}(\mathbf{B})$ into $M_{n}(\mathbf{B}(\mathbf{H}))$.

Since $\phi=F \odot G: M_{n}(\mathbf{U}) \odot M_{n}(\mathbf{L}) \rightarrow M_{n}(\mathbf{B}(\mathbf{H}))$, by applying Theorem 3 and Lemma 3, there are representations $\pi: \mathbf{A} \rightarrow \mathbf{B}\left(\mathbf{H}_{1}\right), \quad \rho: \mathbf{B} \rightarrow \mathbf{B}\left(\mathbf{H}_{2}\right)$, unit vectors $\xi=\left(\xi_{1}, \cdots, \xi_{n}\right) \in \mathbf{H}_{2} \oplus \cdots \oplus \mathbf{H}_{2}$,

$\eta=\left(\eta_{1}, \cdots, \eta_{n}\right) \in \mathbf{H}_{1} \oplus \cdots \oplus \mathbf{H}_{1}$, and $T=\left(T_{i j}\right): \mathbf{H}_{2} \oplus \cdots \oplus \mathbf{H}_{2} \rightarrow \mathbf{H}_{1} \oplus \cdots \oplus \mathbf{H}_{1}$, such that

$$
\phi\left(\left(u_{i j}\right) \otimes\left(l_{i j}\right)\right)=\left\langle\left(\pi\left(u_{i j}\right)\right)\left(T_{i j}\right)\left(\rho\left(l_{i j}\right)\right) \xi, \eta\right\rangle
$$

for $\left(u_{i j}\right) \in M_{n}(\mathbf{U}), \quad\left(l_{i j}\right) \in M_{n}(\mathbf{L})$. 
The element $V \in M_{n}(\mathbf{U}) \otimes_{h} M_{n}(\mathbf{L})$ can be represented using only a finite number of vectors $u_{1}, \cdots, u_{s} \in \mathbf{U}$ and $l_{1}, \cdots, l_{k} \in \mathbf{L}$. Define $\mathbf{J}_{1}=\operatorname{span}\left[\eta_{j}, \pi\left(u_{i}^{*}\right) \eta_{j}\right]$ and $\mathbf{J}_{2}=\operatorname{span}\left[\xi_{j}, \rho\left(l_{i}\right) \xi_{j}\right]$. Choose a separable Hilbert space $\mathbf{H}$ and norm one operators $Y: \mathbf{H}_{1} \rightarrow \mathbf{H}, Z: \mathbf{H}_{2} \rightarrow \mathbf{H}$ which are isometric on $\mathbf{J}_{1}$ and $\mathbf{J}_{2}$ and define completely bounded maps $f: \mathbf{U} \rightarrow \mathbf{B}(\mathbf{H}), g: \mathbf{L} \rightarrow \mathbf{B}(\mathbf{H})$ by

$$
\begin{aligned}
& f(u)=Y \pi(u) Y^{*} ; \\
& g(l)=Y S \rho(l) Z^{*} .
\end{aligned}
$$

Since

$$
\begin{aligned}
\left|F\left(u_{i j}\right)\right|\left|G\left(l_{i j}\right)\right| & \geq\left|(f \otimes g)_{n}(U)\right|=|F \odot G(V)|=\left\langle F \odot G(V) D_{1}, D_{2}\right\rangle \\
& =\left\langle\left(\pi\left(u_{i j}\right)\right)\left(T_{i j}\right)\left(\rho\left(l_{i j}\right)\right) \xi, \eta\right\rangle .
\end{aligned}
$$

It follows that $f$ and $g$ are well-defined and we have

$$
(F \odot G)(V)=\left\langle\left(\pi\left(u_{i j}\right)\right)\left(T_{i j}\right)\left(\rho\left(l_{i j}\right)\right) \xi, \eta\right\rangle
$$

This shows that $F \odot G=\phi$. This completes the sufficiency proof.

\section{A.2. Proof of Theorem 7}

Proof. To avoid technical complications, we only discuss the case $n=2$. In the other words, consider the multivariate switched linear systems with only two subsystems. The calculations are in the same spirit for general $n \in N$ subsystems.

Necessity of proof: Let $\phi: M_{n}(\mathbf{U}) \odot M_{n}(\mathbf{L}) \rightarrow M_{n}(\mathbf{B}(\mathbf{H})), \quad \phi \neq 0$ be completely bounded. By Proposition 5, there are representations $\pi$ of $\mathbf{U}$ on a Hilbert space $\mathbf{H}_{1}$ and $\rho$ of $\mathbf{L}$ on a Hilbert space $\mathbf{H}_{2}$, a bounded linear operator $V_{1}: \mathbf{H} \rightarrow \mathbf{H}_{1}$ with $\mathbf{H}_{1}=\left[\pi(\mathbf{U}) V_{1} \mathbf{H}\right]$ and $V_{2}: \mathbf{H} \rightarrow \mathbf{H}_{2}$ with $\mathbf{H}_{2}=\left[\rho(\mathbf{L}) V_{2} \mathbf{H}\right]$, and a logical projection $T$ from $\mathbf{B}\left(\mathbf{H}_{1}\right)$ into $\mathbf{B}\left(\mathbf{H}_{2}\right)$ such that

$$
\phi(u, l)=V_{1}^{*} \pi(u) T \rho(l) V_{2}
$$

for all $u \in \mathbf{U}, \quad l \in \mathbf{L}$, with $\left\|V_{1}^{*}\right\|=\|F\|,\left\|V_{2}\right\|=\|G\|=1,\left\|T\left(H_{1}\right)\right\|_{c b}=1$. Let $\eta_{0}$ be a unit vector in $\mathbf{H}_{2}$ such that $T_{\mathbf{H}_{2}}=\left[T \rho(\mathbf{L}) V_{2} \mathbf{H}\right]=\operatorname{span}\left\{\eta_{0}\right\}$ and let $\left\{e_{1}=\left[\begin{array}{l}1 \\ 0\end{array}\right], e_{2}=\left[\begin{array}{l}0 \\ 1\end{array}\right]\right\}$ be the standard basis for $\mathbf{H}$. For $i, j=1,2$, there are logical functional $g_{i}$ on $\mathbf{H}_{1}$ such that

$$
g_{j}(l) \eta_{0}=T \rho(l) V_{2} e_{j}
$$

Let $\xi_{0}$ be a unit vector in $\mathbf{H}$ such that $\mathbf{H}_{1}=\left[\pi(\mathbf{U}) V_{1} \mathbf{H}\right]=\operatorname{span}\left\{\xi_{0}\right\}$ and the linear functional $f_{i}$ on $\mathbf{U}$ such that

$$
f_{i}(u) \xi_{0}=\pi(u) V_{1} e_{i}
$$

for all $u \in \mathbf{U}, l \in \mathbf{L}$. Then we have

$$
\begin{aligned}
\left\langle\phi(u, l)\left[\begin{array}{l}
a_{1} \\
a_{2}
\end{array}\right],\left[\begin{array}{l}
b_{1} \\
b_{2}
\end{array}\right]\right\rangle & =\sum_{i, j=1}^{2} b_{i} a_{j}\left\langle\phi(u, l) e_{j}, e_{i}\right\rangle \\
& =\sum_{i, j=1}^{2} b_{i} a_{j}\left\langle T \rho(l) V_{2} e_{j}, \pi\left(u^{*}\right) V_{1} e_{i}\right\rangle \\
& =\sum_{i, j=1}^{2} b_{i} a_{j}\left\langle g_{j}(l) \eta_{0}, f_{i}\left(u^{*}\right) \xi_{0}\right\rangle \\
& =\sum_{i, j=1}^{2} b_{i} a_{j} g_{j}(l) f_{i}\left(u^{*}\right) \\
& =\left\langle\left[\begin{array}{cc}
f_{1}^{*}(u) & 0 \\
f_{2}^{*}(u) & 0
\end{array}\right]\left[\begin{array}{cc}
g_{1}(l) & g_{2}(l) \\
0 & 0
\end{array}\right]\left[\begin{array}{l}
a_{1} \\
a_{2}
\end{array}\right],\left[\begin{array}{l}
b_{1} \\
b_{2}
\end{array}\right]\right\rangle .
\end{aligned}
$$


for all $u \in \mathbf{U}, l \in \mathbf{L}$ and for all $a_{i}, b_{i} \in \mathbf{H},(i=1,2)$. This implies that $\phi=F \odot G$, where $F=\left(\begin{array}{cc}f_{1} & f_{2} \\ 0 & 0\end{array}\right)$ and $G=\left(\begin{array}{cc}g_{1} & g_{2} \\ 0 & 0\end{array}\right)$. It follows from Lemma 4 that $F: M_{2}(\mathbf{U}) \rightarrow M_{2}(\mathbf{B}(\mathbf{H}))$ and $G: M_{2}(\mathbf{L}) \rightarrow M_{2}(\mathbf{B}(\mathbf{H}))$ (Here, $\mathbf{L}=\mathbf{H}_{2}$ ) are completely bounded with $\|\phi\|_{c b}=\inf \left\{\|F\|_{c b}\right\}$. This completes the necessity proof.

Sufficiency of proof: It follows, from Lemma 4 , that the tensor-product representation $\phi$ is completely bounded from $M_{n}(\mathbf{U}) \odot M_{n}(\mathbf{L})$ into $M_{n}(\mathbf{B}(\mathbf{H}))$ with $\inf \left\{\|F\|_{c b}\right\}=\|\phi\|_{c b}$. It suffices to show that the associated bilinear representation $V$ is completely bounded from $M_{n}(\mathbf{U}) \times M_{n}(\mathbf{L})$ into $M_{n}(\mathbf{B}(\mathbf{H}))$. Here we only prove the case $n=2$ as in the necessity proof.

Since $\phi: M_{2}(\mathbf{U}) \odot M_{2}(\mathbf{L}) \rightarrow M_{2}(\mathbf{B}(\mathbf{H}))$, by Theorem 3, there are representations $\pi$ of $\mathbf{U}$ on a Hilbert space $\mathbf{H}_{1}$ and $\rho$ of $\mathbf{L}$ on a Hilbert space $\mathbf{H}_{2}$, a bounded linear operator $V_{1}: \mathbf{H} \rightarrow \mathbf{H}_{1}$ with $\mathbf{H}_{1}=\left[\pi(\mathbf{U}) V_{1} \mathbf{H}\right]$ and $V_{2}: \mathbf{H} \rightarrow \mathbf{H}_{2}$ with $\mathbf{H}_{2}=\left[\rho(\mathbf{L}) V_{2} \mathbf{H}\right]$, and a logical operator $T$ from $\mathbf{B}\left(\mathbf{H}_{1}\right)$ into $\mathbf{B}\left(\mathbf{H}_{2}\right)$ with $\left\|T\left(H_{1}\right)\right\|_{c b}=1$ such that

$$
\phi(u, l)=V_{1}^{*} \pi(u) T \rho(l) V_{2}
$$

for all $u \in \mathbf{U}, l \in \mathbf{L}$, with $\left\|V_{1}^{*}\right\|=\|F\|,\left\|V_{2}\right\|=\|G\|=1$.

Since very element $\xi \in \pi(\mathbf{U}) V_{1} \mathbf{H}$ can be written as

$$
\xi=\sum_{i=1}^{2} \pi\left(u_{i}\right) V_{1} e_{i}
$$

for some $u_{i} \in \mathbf{U}, \quad(i=1,2)$, we define a linear functional $\tilde{f}: \pi(\mathbf{U}) V_{1} \mathbf{H} \rightarrow \mathbf{B}(\mathbf{H})$ by

$$
\hat{f}\left(\sum_{i=1}^{2} \pi\left(u_{i}\right) V_{1} e_{i}\right)=\sum_{i=1}^{2} f_{i}\left(u_{i}\right)
$$

for all $\sum_{i=1}^{2} \pi\left(u_{i}\right) V_{1} e_{i} \in \pi(\mathbf{U}) V_{1} \mathbf{H}$.

And very element $\eta \in \rho(\mathbf{L}) V_{2} \mathbf{H}$ can be written as

$$
\eta=\sum_{j=1}^{2} \rho\left(l_{j}\right) V_{2} e_{j}
$$

for some $l_{j} \in \mathbf{L}, \quad(j=1,2)$, we define a linear functional $\tilde{g}: \rho(\mathbf{L}) V_{2} \mathbf{H} \rightarrow \mathbf{B}(\mathbf{H})$ by

$$
\hat{g}\left(\sum_{i=1}^{2} \rho\left(l_{j}\right) V_{2} e_{j}\right)=\sum_{j=1}^{2} g_{j}\left(l_{j}\right)
$$

for all $\sum_{j=1}^{2} \rho\left(l_{j}\right) V_{2} e_{j} \in \rho(\mathbf{L}) V_{2} \mathbf{H}$. Since

$$
\begin{aligned}
\left|\sum_{i=1}^{2} f_{i}\left(u_{i}\right)\right|\left|\sum_{j=1}^{2} g_{j}\left(l_{j}\right)\right| & =\sum_{i, j=1}^{2} f_{i}\left(u_{i}^{*}\right) g_{j}\left(l_{j}\right) \\
& \geq\left\langle\left[\begin{array}{cc}
F^{*}\left(u_{1}^{*}\right) & 0 \\
F^{*}\left(u_{2}^{*}\right) & 0
\end{array}\right]\left[\begin{array}{cc}
G\left(l_{1}\right) & G\left(l_{2}\right) \\
0 & 0
\end{array}\right]\left[\begin{array}{l}
e_{1} \\
e_{2}
\end{array}\right],\left[\begin{array}{l}
e_{1} \\
e_{2}
\end{array}\right]\right\rangle \\
& =\left\langle\phi_{2}\left(\left[\begin{array}{ll}
u_{1}^{*} & 0 \\
u_{2}^{*} & 0
\end{array}\right],\left[\begin{array}{ll}
l_{1} & l_{2} \\
0 & 0
\end{array}\right]\right)\left[\begin{array}{l}
e_{1} \\
e_{2}
\end{array}\right],\left[\begin{array}{c}
e_{1} \\
e_{2}
\end{array}\right]\right\rangle \\
& =\left\langle\left(T \otimes I_{2}\right) \rho\left(\left[\begin{array}{cc}
l_{1} & l_{2} \\
0 & 0
\end{array}\right]\right) U_{2}, \pi\left(\left[\begin{array}{cc}
u_{1} & l_{2} \\
0 & 0
\end{array}\right]\right) U_{1}\right\rangle \\
& =\left\langle T \sum_{j=1}^{2} \rho\left(l_{j}\right) V_{2} e_{j}, \sum_{i=1}^{2} \pi\left(u_{i}^{*}\right) V_{1} e_{i}\right\rangle .
\end{aligned}
$$

where $U_{2}=\left(V_{2} \otimes I_{2}\right)\left[\begin{array}{l}e_{1} \\ e_{2}\end{array}\right], U_{1}=\left(V_{1} \otimes I_{2}\right)\left[\begin{array}{l}e_{1} \\ e_{2}\end{array}\right]$, it follows that $\tilde{f}$ and $\tilde{g}$ are well-defined and we have 


$$
\begin{aligned}
\left|\hat{f}\left(\sum_{i=1}^{2} \pi\left(u_{i}\right) V_{1} e_{i}\right)\right|\left|\hat{g}\left(\sum_{j=1}^{2} \rho\left(l_{i}\right) V_{2} e_{i}\right)\right| & =\left|\sum_{i=1}^{2} f_{i}\left(u_{i}\right)\right|\left|\sum_{j=1}^{2} g_{j}\left(l_{j}\right)\right| \\
& =\left\langle T \sum_{j=1}^{2} \rho\left(l_{j}\right) V_{2} e_{j}, \sum_{i=1}^{2} \pi\left(u_{i}^{*}\right) V_{1} e_{i}\right\rangle .
\end{aligned}
$$

lead to

$$
\hat{f}(\eta) \hat{g}(\zeta)=\langle T \eta, \zeta\rangle
$$

for all $\eta, \zeta \in \mathbf{H}$. Therefore,

$$
\begin{aligned}
\|\hat{\phi}\|=\|\hat{f} \otimes \hat{g}\| & \leq\left|\hat{f}\left(\sum_{i=1}^{2} \pi\left(u_{i}\right) V_{1} e_{i}\right)\right|\left|\hat{g}\left(\sum_{j=1}^{2} \rho\left(l_{j}\right) V_{2} e_{j}\right)\right| \\
& \leq\left|\left\langle T \sum_{j=1}^{2} \rho\left(l_{j}\right) V_{2} e_{j}, \sum_{i=1}^{2} \pi\left(u_{i}^{*}\right) V_{1} e_{i}\right\rangle\right|,
\end{aligned}
$$

where $\left\|\sum_{i=1}^{2} \pi\left(u_{i}\right) V_{1} e_{i}\right\| \leq 1,\left\|\sum_{j=1}^{2} \rho\left(l_{j}\right) V_{2} e_{j}\right\|=1$.

Since $\pi(\mathbf{U}) V_{1} \mathbf{H}$ is a dense subspace of $\mathbf{H}$, there is a unique norm preserving linear extension of $\tilde{f}$ from $\pi(\mathbf{U}) V_{1} \mathbf{H}$ to the whole Hilbert space $\mathbf{H}=\left[\pi(\mathbf{U}) V_{1} \mathbf{H}\right]$, still denoted by $\tilde{f}$. It is shown that $F \odot G=\phi$ with $\inf \left\{\|F\|_{c b}\right\}=\|\phi\|_{c b}$. This completes the sufficiency proof.

\section{A.3. Proof of Theorem 10}

Proof. We first consider the case $n=3$. By Proposition $1, \mathbf{E}_{2} \otimes_{h} \mathbf{E}_{3}$ is completely isometrically isomorphic to a subspace $\mathbf{F}$ of some $C^{*}$-algebra $\varphi$. The linear map $\phi: \mathbf{E}_{1} \otimes_{h} \mathbf{E}_{2} \otimes_{h} \mathbf{E}_{3} \rightarrow \mathbf{B}(\mathbf{H})$ is completely bounded, which corresponds to a trilinear map $V$ from Proposition 2. We identify $\mathbf{E}_{2} \otimes_{h} \mathbf{E}_{3}$ with $\mathbf{F}$, then the map $\phi: \mathbf{E}_{1} \otimes_{h} \mathbf{F} \rightarrow \mathbf{B}(\mathbf{H})$ is completely bounded. Thus, by the bilinear case there exist Hilbert spaces $\mathbf{B}\left(\mathbf{H}_{1}\right)$ and $\mathbf{B}\left(\mathbf{H}_{2}\right)$, representations $\pi_{1}: \mathbf{U}_{1} \rightarrow \mathbf{B}\left(\mathbf{H}_{1}\right)$ and $\rho: \varphi \rightarrow \mathbf{B}\left(\mathbf{H}_{2}\right)$ isometries $V_{1}: \mathbf{B}(\mathbf{H}) \rightarrow \mathbf{B}\left(\mathbf{H}_{1}\right)$, $W: \mathbf{B}(\mathbf{H}) \rightarrow \mathbf{B}\left(\mathbf{H}_{2}\right)$, and a contraction $T: \mathbf{B}\left(\mathbf{H}_{2}\right) \rightarrow \mathbf{B}\left(\mathbf{H}_{1}\right)$, such that

$$
\phi\left(u_{1} \otimes f\right)=V_{1} * \pi\left(u_{1}\right) T \rho(f) W
$$

Since the inclusion $\varsigma: \mathbf{E}_{2} \otimes_{h} \mathbf{E}_{3} \rightarrow \mathbf{F}$ is completely bounded, the map $\psi=\rho \circ \varsigma: \mathbf{E}_{2} \otimes_{h} \mathbf{E}_{3} \rightarrow \mathbf{B}\left(\mathbf{H}_{2}\right)$ is completely bounded. Applying the representation theorem 4 for completely bounded bilinear maps to the map $\psi$ and substituting this expression into the formula for $\phi: \mathbf{E}_{1} \otimes_{h} \mathbf{F} \rightarrow \mathbf{B}(\mathbf{H})$, then we can get $\phi=F_{1} \odot F_{2} \odot F_{3}$.

From Lemma 2, we can obtain $\|\phi\|_{c b} \leq\left\|F_{1}\right\|_{c b}\left\|F_{2}\right\|_{c b}$. Then, for the map $\psi=\rho \circ \varsigma: \mathbf{E}_{2} \otimes_{h} \mathbf{E}_{3} \rightarrow \mathbf{B}\left(\mathbf{H}_{2}\right)$ combining with inclusion $\varsigma: \mathbf{E}_{2} \otimes_{h} \mathbf{E}_{3} \rightarrow \mathbf{F}$, we have $\|\psi\|_{c b} \leq\left\|F_{2}\right\|_{c b}\left\|F_{3}\right\|_{c b}$. Consequently, by substituting this expression into the formula for $\phi: \mathbf{E}_{1} \otimes_{h} \mathbf{F} \rightarrow \mathbf{B}(\mathbf{H})$, we can obtain $\|\phi\|_{c b} \leq\left\|F_{1}\right\|_{c b}\left\|F_{2}\right\|_{c b}\left\|F_{3}\right\|_{c b}$. When $n>3$, the results can be directly derived as the same line of this idea. 